\title{
AVISOS DE FENÓMENOS METEOROLÓGICOS ADVERSOS (FMA)
}

\section{Jesús Gordaliza Borrego}

Delegación Territorial de AEMET en Castilla y León, C/ Orión 1 Valladolid, jgordalizab@aemet.es

\section{1.- Entorno legal}

El Real Decreto 186/2008 de 8 de febrero aprueba el Estatuto de la Agencia Estatal de Meteorología (AEMET).

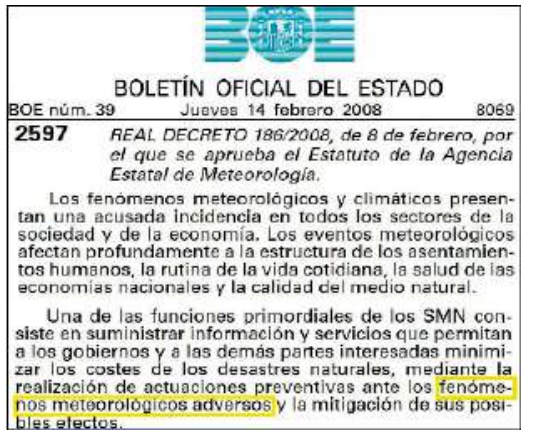

Fig. 1.- El Real Decreto 186/2008

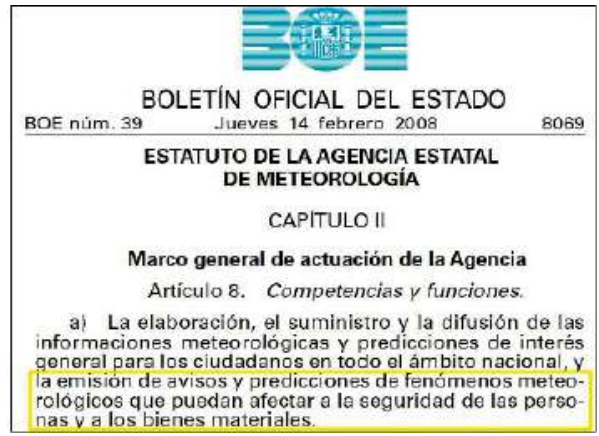

Fig. 2.- Artículo 8 de competencias y funciones de AEMET

Una de las competencias y funciones de la AEMET es la de emitir avisos y predicciones de fenómenos meteorológicos adversos (FMA).

\section{2.- Unidades de FMA en el Sistema Nacional de Predicción}

El Sistema Nacional de Predicción de AEMET está organizado en cuanto a FMA en 8 unidades operativas.

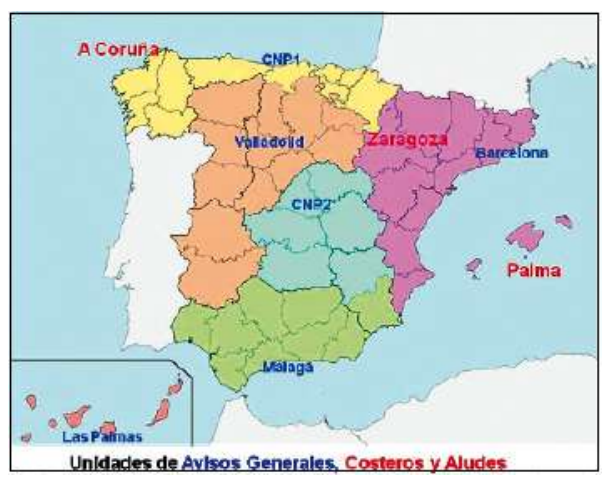

Dependiendo de los FMA, estas unidades son:

- 5 unidades de avisos generales: CNP (Centro Nacional de Predicción) y Grupos de Predicción y Vigilancia en Valladolid, Málaga, Barcelona y Las Palmas. - 2 unidades de avisos costeros: A Coruña y Palma.

- 1 unidad de aviso de aludes: Zaragoza.

Fig. 3.- Unidades operativas de FMA en AEMET 


\section{3.- Meteoalerta}

Meteoalerta es el principal documento operativo por el que se rige la emisión de FMA en AEMET.

En el mismo está incluido toda la operatividad de tipo de FMA y su emisión desde las unidades operativas.

Meteoalerta está consensuado entre AEMET y Protección Civil.

Se puede consultar en la web de AEMET en el siguiente enlace: http://www.aemet.es/documentos/es/eltiempo/prediccion/avisos/plan_meteoalerta_v6.pdf

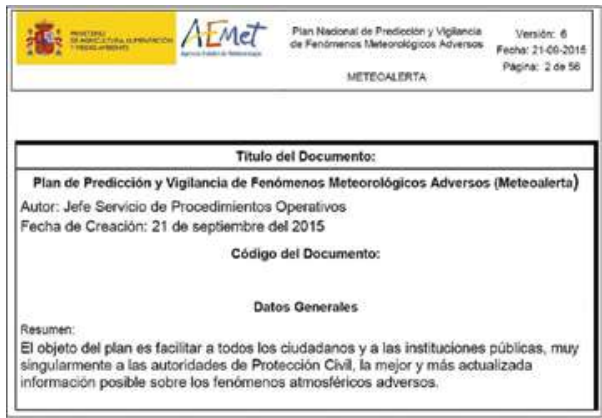

Fig. 5.- Código de colores de los FMA
Fig. 4.- Meteoalerta

Los FMA tienen el código semafórico de colores; ver siguiente figura:

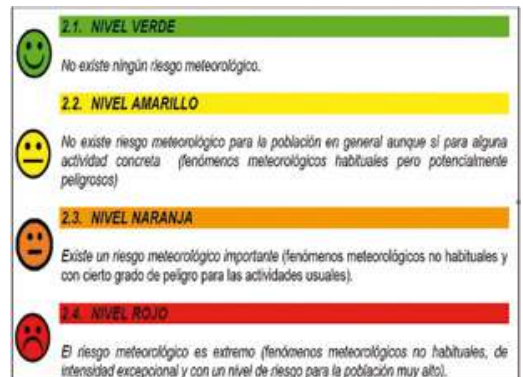

Hay que distinguir claramente entre avisos y alertas: los avisos los emite AEMET y las alertas las emite Protección Civil o la administración correspondiente (por ejemplo un ayuntamiento).

\section{4.- Tipos de FMA}

Los fenómenos meteorológicos que pueden motivar la emisión de un FMA son los siguientes:

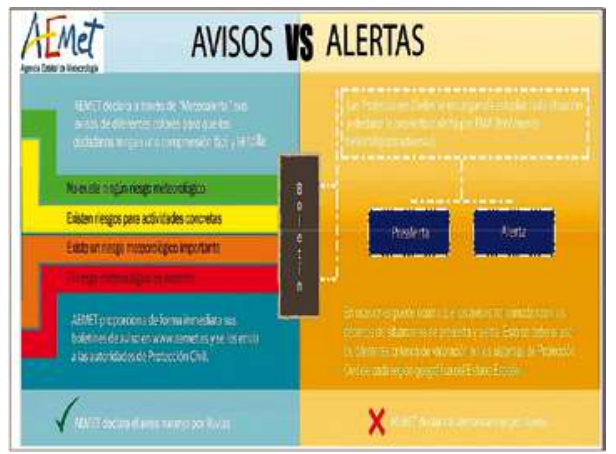

- Lluvias con acumulaciones importantes en 1 hora 012 horas.

- Nevadas con acumulaciones importantes en el suelo en 24 horas.

- Rachas de viento en $\mathrm{km} / \mathrm{h}$.

- Tormentas: ocurrencia y grado de intensidad.

- Temperaturas máximas.

- Temperaturas mínimas.

- Fenómenos costeros: viento y altura de olas.

Fig. 6.- Distinción entre aviso y alerta 
- Aludes.

- Polvo en suspensión: visibilidad en metros.

- Galerna en el área cantábrica y norte de Galicia: ocurrencia e intensidad.

- Rissagues o risagas en Baleares: oscilación del nivel del mar.

- Niebla.

- Deshielo.

\section{5.- Zonas de FMA}

El territorio español está dividido en 182 zonas de FMA.

Esta división se ha realizado en consenso entre AEMET y Protección Civil dependiendo de la situación geográfica y orografía.

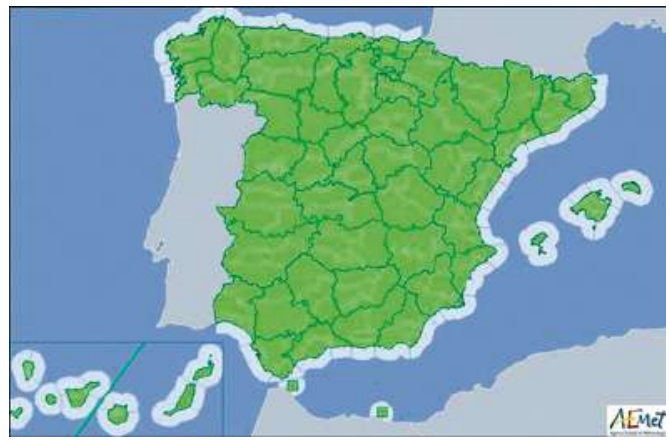

Fig. 7.- Zonas de FMA

\section{6.- Umbrales de FMA}

Hay FMA que tienen distintos umbrales por los que se va escalando en su código semafórico de colores.

Estos fenómenos atmosféricos concretos son: temperaturas máximas y mínimas, vientos, precipitaciones en 1 o 12 horas y nevadas.

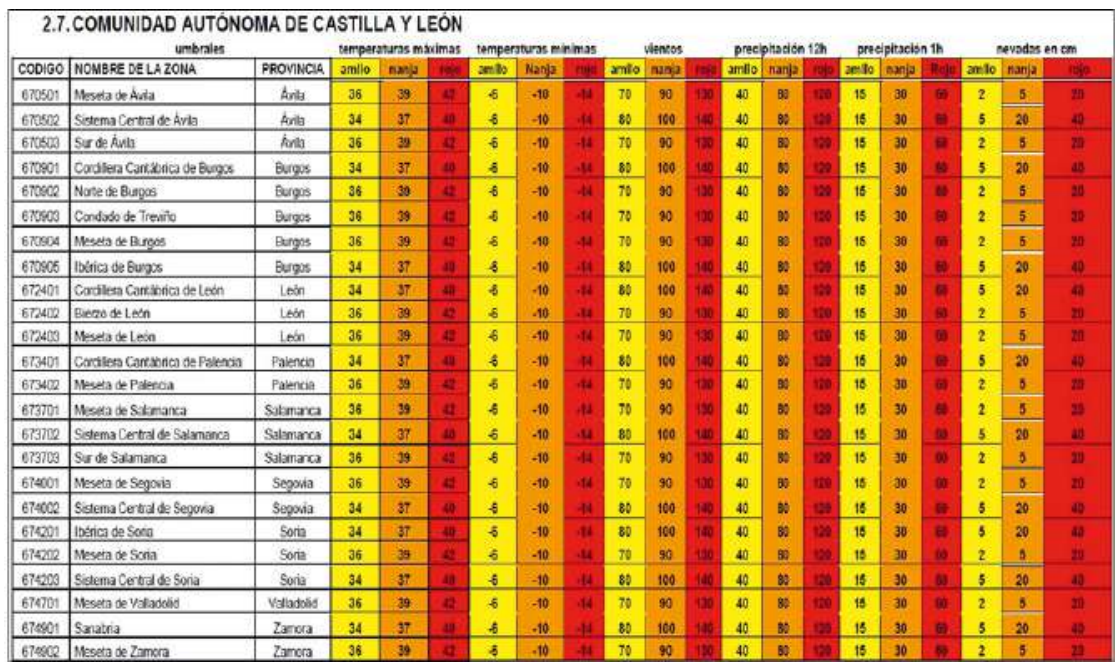

Fig. 8.- Umbrales de FMA en las zonas de aviso de Castilla y León 


\begin{tabular}{|c|c|c|c|c|c|c|c|c|c|c|c|c|c|c|c|c|c|c|c|c|}
\hline \multicolumn{21}{|c|}{ 2.5. COMUNIDAD AUTONOMA DE CANARIAS } \\
\hline & \multicolumn{2}{|c|}{ unbrales } & \multicolumn{3}{|c|}{ cengermens módinnas } & \multirow{2}{*}{\multicolumn{3}{|c|}{ temperaturas mivimes }} & \multirow{2}{*}{\multicolumn{2}{|c|}{$\begin{array}{c}\text { vientos } \\
\text { [amile I naravia }\end{array}$}} & \multicolumn{4}{|c|}{ poceciphacien $12 \mathrm{~h}$} & \multicolumn{3}{|c|}{ precipitacion th } & \multicolumn{3}{|c|}{ nerrodes en om } \\
\hline c00130 & NOWDER CE LAZONA & PROMUIICIA & anlis. & maranta & $n$ & & & & & & nat & amta & harrial & 曋象 & amble] & mearia & $m$ & ants & inararia & mais \\
\hline \begin{tabular}{|l|l|}
050001 \\
\end{tabular} & Noted de Oranconves & LnPalne & 24 & $\pi$ & +2 & 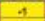 & 4 & $\rightarrow$ & no & 90 & 123 & 46 & s6. & 123 & is & 30 & u & 2 & 8 & 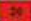 \\
\hline \begin{tabular}{|c|c|}
50003 \\
\end{tabular} & Cuntones de Gren Canera & Las Painos & 24 & 37 & 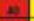 & 4 & 4 & 1 & 70 & se & 131 & 46 & 10 & as & 18 & 30 & is & 2 & 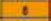 & 3 \\
\hline 650004 & 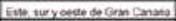 & LatPames & 34 & $x$ & $\infty$ & $\rightarrow$ & 4 & + & $n$ & es & 139 & 46 & in & (2) & is & 10 & $u$ & 2 & $s$ & 90 \\
\hline \begin{tabular}{|c|}
69101 \\
\end{tabular} & Lanzmon & Las Fings & 34 & 37 & 4 & $A$ & 4 & + & No & * & 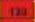 & 4 & 10 & 10 & is & 30 & a & 2 & 8 & st \\
\hline \begin{tabular}{|c|c|}
0.5201 \\
\end{tabular} & Fuerterenura & Las falmes & 24 & $\pi$ & 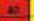 & $\rightarrow$ & 4 & $*$ & $\pi$ & s. & 23 & 4 & D. & $\infty$ & 18 & 39 & e & 2 & $s$ & 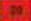 \\
\hline excser & Cantres de bis Pina & Sucher co Terivite & 34 & 37 & as & 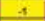 & 4 & 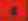 & $x_{0}-1-1-1-1$ & e & a & 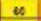 & 100 & (2) & 16 & 30 & is & 2 & 6 & $x$ \\
\hline \begin{tabular}{|c|}
658303 \\
\end{tabular} & Ested de la Faina & Sta Cinz de Tenerte & 34 & 표 & si. & $\rightarrow$ & 4 & + & 70 & s & 19 & se & 100 & ws & is & 30 & $*$ & 2 & 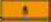 & se \\
\hline 068004 & cenedebasus & Suchzoe Tenerte. & 34 & 7 & $\infty$ & 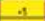 & 4 & $\&$ & $\pi 0$ & $\rightarrow 0$ & 131 & $\omega$ & 100 & 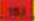 & 18 & 30 & e & 2 & 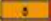 & 2 \\
\hline \begin{tabular}{|c|}
0.5901 \\
\end{tabular} & LSTonea & Suchuco-Tereif & 34 & 97 & 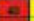 & 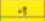 & 4 & s & 70 & 9 & {$[a]$} & ot & 100. & 09 & 10 & 30 & s & 2 & $a$ & $a$ \\
\hline excsor & GHOSO & Su Che do Tenerto & 24 & $n$ & 4) & $A$ & 4 & $t$ & 30 & $\phi$ & $a x$ & $\Leftrightarrow$ & 100 & 103 & s6 & 20 & ut & 2 & 1 & se \\
\hline \begin{tabular}{|l|}
650601 \\
\end{tabular} & Noste de Tenimb & Su Cine of Tenerte & 34 & $x$ & $\$$ & 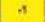 & 4 & + & 70 & so & 13i & 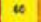 & 100 & ins & 15 & 10 & te & 2 & 8 & al \\
\hline \begin{tabular}{|c|}
$x 0002$ \\
\end{tabular} & Avea Horegediana on Terenth & Sta Chu of Temerte & 34 & 37 & $\approx$ & 4 & 4 & $*$ & no & 90 & 120 & $\omega$ & 100 & 15 & 16 & 10 & o & 2 & 8 & 36 \\
\hline \begin{tabular}{|l|}
$0 t 8003$ \\
\end{tabular} & Este sur yueste is Teneifte & Su Chiz de Tererite & 94 & 37 & 28 & 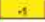 & 4 & 党 & 70 & $x$ & in & e & 100 & $\cos$ & 15 & 30 & is & 2 & 5 & a \\
\hline
\end{tabular}

Fig. 9.- Umbrales de FMA en las zonas de aviso de Canarias

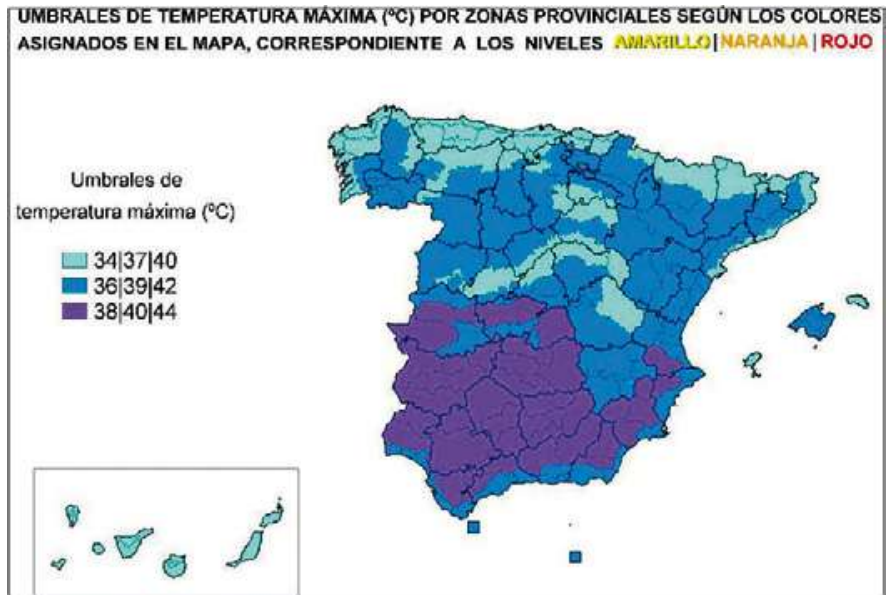

Los umbrales van a depender de la orografía y situación geográfica de cada zona concreta.

$\checkmark$ Umbrales de las temperaturas máximas y mínimas:

Disminuyen con la latitud y la altitud.

Fig. 10.- Umbrales para temperatura máxima
Fig. 11.- Umbrales para temperatura mínima

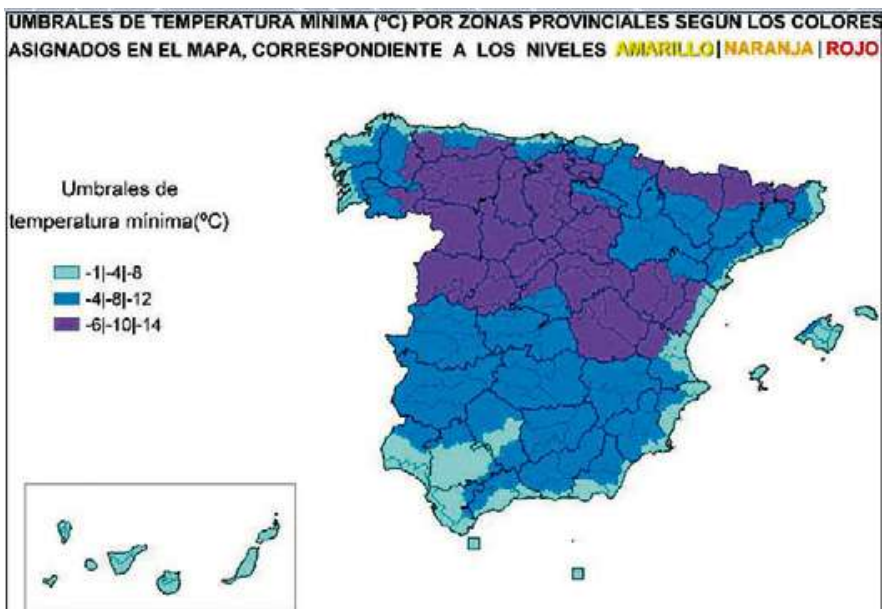




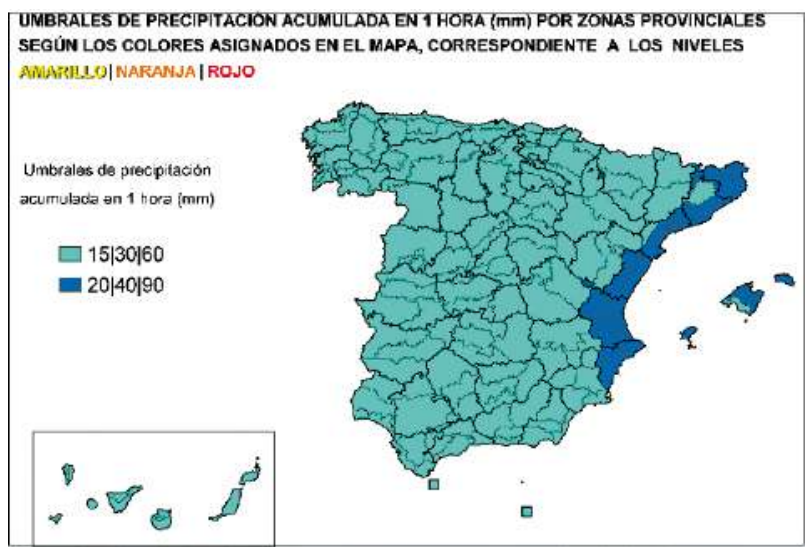

\section{$\checkmark$ Umbrales de pre- cipitación acumulada en 1 y 12 horas}

Aumenta en el litoral mediterráneo por la mayor ocurrencia de precipitaciones muy fuertes en esta región.

Fig. 12.- Umbrales para precipitación acumulada en 1 hora

Fig. 13.- Umbrales para precipitación acumulada en

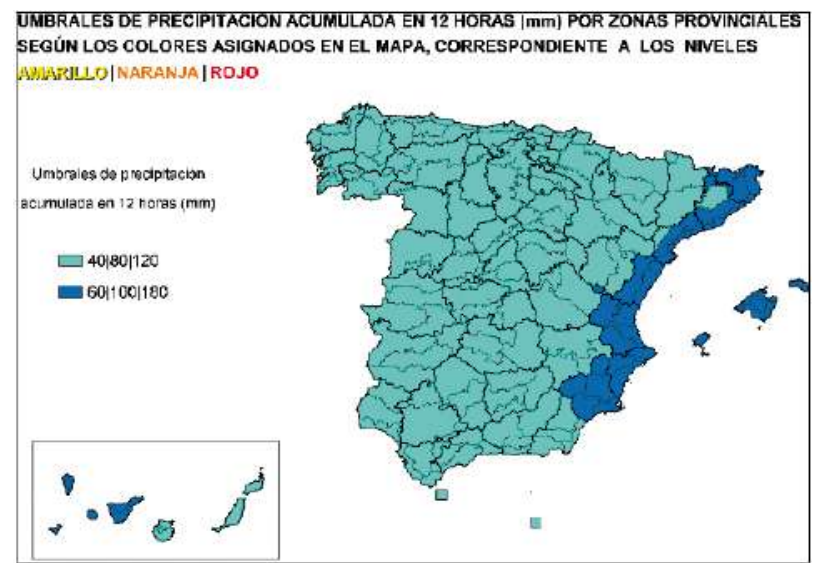
12 horas

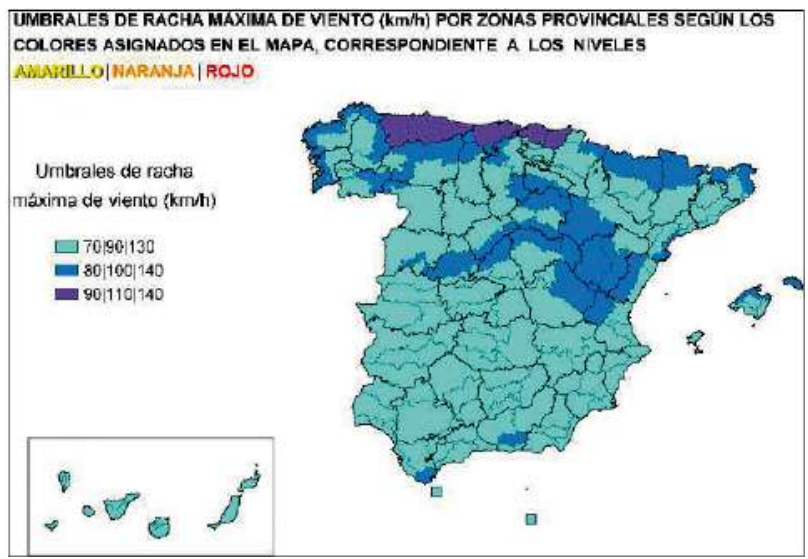

\author{
$\checkmark$ Umbrales de ra- \\ chas de viento: \\ Aumenta con la \\ altitud y en áreas del \\ Cantábrico por la mayor \\ ocurrencia de rachas \\ muy fuertes en estas \\ zonas.
}

Fig. 14.- Umbrales para rachas de viento 
$\checkmark$ Umbrales de nieve acumulada en 24 horas.

Aumenta con la altitud.

Fig. 15.- Umbrales para nieve

\section{7.- Avisos por tormenta}

Dependen de varios factores:

* Si las tormentas son generalizadas y organizadas.

* Intensidad de las rachas de viento asociadas.

* Tamaño del granizo en su caso.

* Probabilidad de ocurrencia de tornado.

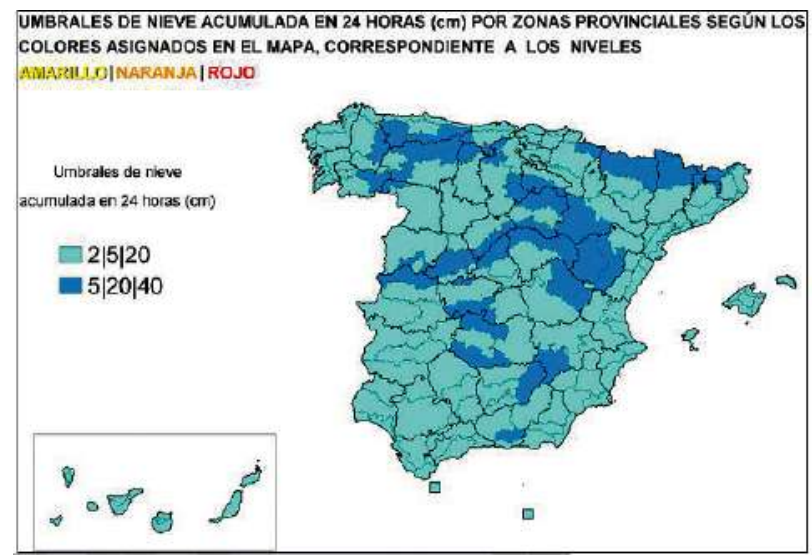

\begin{tabular}{|c|c|c|c|}
\hline Zonas & Annarillo & Naranja & Rojo \\
\hline $\begin{array}{l}\text { CANTÁBRICAS } \\
\text { ATLÁNTICAS }\end{array}$ & 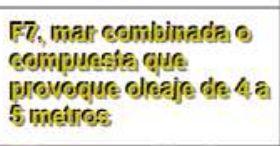 & $\begin{array}{l}\text { Fa y Fa } \\
\text { mar combinadia } \\
\text { compuesta gue } \\
\text { proverque oleaje de } 5 \text { a } \\
\text { 8 motres }\end{array}$ & $\begin{array}{l}\text { A partir de Fia } \\
\text { mar combinada o } \\
\text { compuesta que } \\
\text { provoque oleaje de } \\
\text { mals de a metros }\end{array}$ \\
\hline MEDITERRÁNEAS & 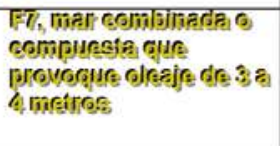 & 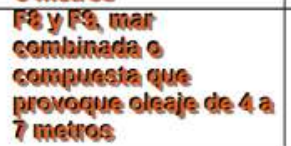 & $\begin{array}{l}\text { A partir de Fia. } \\
\text { mar combinadas } \\
\text { compuesta que } \\
\text { provoque oleaje de } \\
\text { miss de } 7 \text { meetros }\end{array}$ \\
\hline
\end{tabular}

\section{8.- Avisos por fenómenos costeros}

Fig. 17.- Avisos por fenómenos costeros

Dependen de varios factores:

* Fuerza del viento (escala Beaufort)

* Oleaje (escala Douglas)

Se distinguen dos zonas: mediterránea y cantábrica-atlántica 


\section{9.- Avisos por aludes}

Dependen del índice de riesgo y del nivel de salida (altitud en la que la nieve colapsa y comienza a moverse).

\begin{tabular}{|c|c|}
\hline ESCALA DE RIESGO DE ALUDES & NIVEL \\
\hline 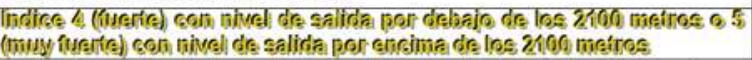 & ALMARIILLO \\
\hline Indice 5 (muy fuerte) sen nivel de salida per debaje de les 2100 metres & WARAIWA \\
\hline $\begin{array}{l}\text { Sluacion excepcional de riesgo generalizado de nivel naranja que } \\
\text { afecte a una anplia zona }\end{array}$ & Rov \\
\hline
\end{tabular}

Fig. 18.- Avisos por aludes

\section{0.- Avisos por galerna}

Una galerna es un viento súbito muy fuerte y racheado, acompañado o no de precipitaciones que suele cortar de manera brusca un tiempo apacible y generalmente caluroso. Es un fenómeno propio del mar Cantábrico donde se desplazan de W a E.

Fig. 19.- Avisos por galerna

\begin{tabular}{|c|c|c|}
\hline ZONAS & NIVEL & CARACTERISTICAS \\
\hline GALICIA & AMARILOO & 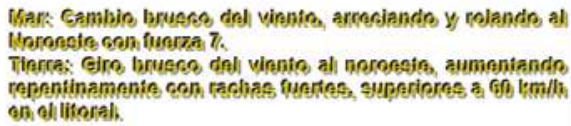 \\
\hline CANTABRIA & NARANSA & 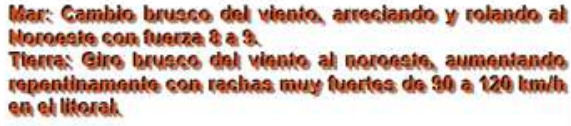 \\
\hline VASCO & RONO & 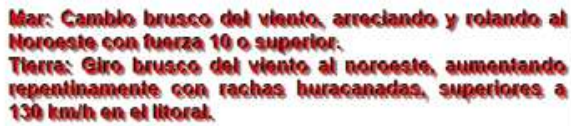 \\
\hline
\end{tabular}

\section{1.- Avisos por risagas}

Oscilación del nivel del mar en puertos, calas o bahías, motivadas por causas meteorológicas en condiciones de resonancia; suelen tener su ocurrencia en las islas Baleares.

\begin{tabular}{|c|c|c|}
\hline ZONAS & NIVEL & CARACTERISTICAS \\
\hline \multirow{3}{*}{$\begin{array}{l}\text { ILLES } \\
\text { BALEARS }\end{array}$} & ASWARILILO & 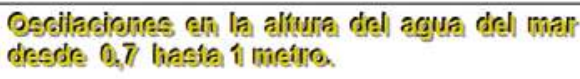 \\
\hline & NARANWA & $\begin{array}{l}\text { Qseillaciones dia la altura del agua diel mar } \\
\text { d'esdie } 1 \text { liasta } 2 \text { metros. }\end{array}$ \\
\hline & RONO & $\begin{array}{l}\text { Oscilaciones superiores a } 2 \text { metros on la } \\
\text { altura del agua del mar. }\end{array}$ \\
\hline
\end{tabular}

Fig. 20.Avisos por risagas 
12.- Avisos por deshielo

\begin{tabular}{|c|c|c|}
\hline ZONAS & NIVEL & CARACTERISTICAS \\
\hline \multirow{3}{*}{$\begin{array}{l}\text { CASTILLA Y } \\
\text { LEÓN, } \\
\text { ARAGÓN, } \\
\text { NAVARRA Y } \\
\text { RIOJA }\end{array}$} & AMARALLO & 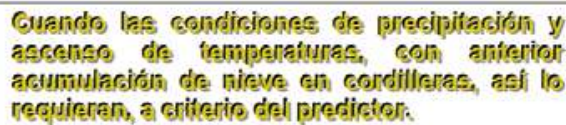 \\
\hline & NARALWA & $\begin{array}{l}\text { Guando las condicionas de precipitacion y } \\
\text { ascenso de temperaturas, sen anterior } \\
\text { acumulacidn de nieve en sordillerae, ael lo } \\
\text { requieran, a griterio del predictor }\end{array}$ \\
\hline & RONO & $\begin{array}{l}\text { Guando las condiciones de presipitación y } \\
\text { ascenso de temperaturas. con anterior } \\
\text { acumulación de nieve en cordilteras, asi lo } \\
\text { requieran, a criterio del predictor. }\end{array}$ \\
\hline
\end{tabular}

Fig. 21.- Avisos por deshielo

\section{3.- Avisos por niebla y polvo en suspensión}

Sólo se emitirán avisos amarillos.

En caso de nieblas se emitirán para reducciones de visibilidad de 200 metros en general.

En el caso de polvo en suspensión se emitirán para reducciones de visibilidad de menos de 3000 metros.

\section{4.- Comunicación de FMA al ciudadano}

Hay varias formas de comunicación directa de los FMA al ciudadano:

* Página web : http://www.aemet.es/es/eltiempo/prediccion/avisos?w=hoy

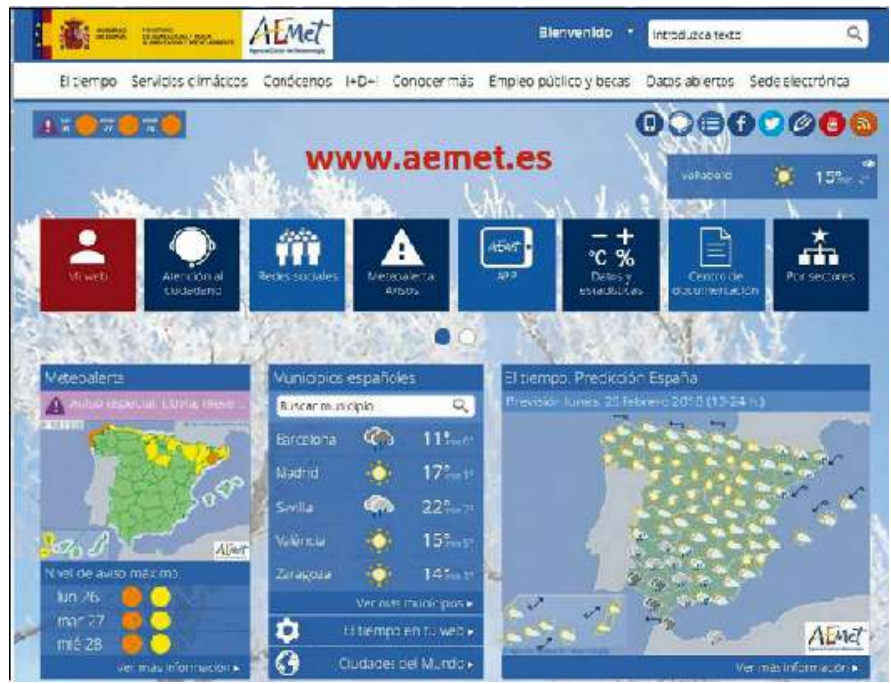

Fig. 22.- página web de AEMET 
* APP para dispositivos móviles

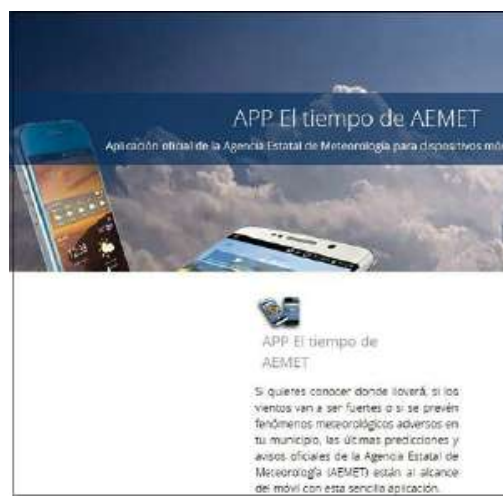

Fig. 23.- APP de AEMET

* Twitter @AEMET_Esp

Fig. 24.- Twitter de @AEMET_Esp

AOWr AEMET \&
26/02 12:01 \#AEMET_Esp
por temp. min y/o nevadas y/o costeros para
mañana en Navarra, C. Valenciana, Galicia y
Cataluña + infoCLICK EN LA IMAGEN o en
aemet.es/es/eltiempo/pr...

aemet.es/es/eltiempo/pr ...

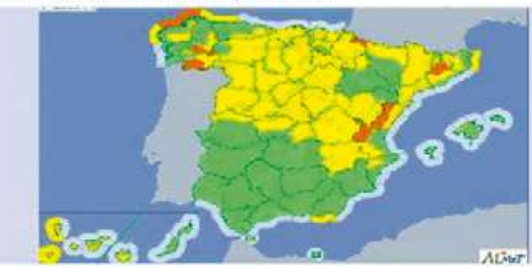

AEMET AVISOS

26/02 12:01 \#AEMET mavisos nivel naranja por temp. min y/o nevadas y/o costeros para maĥana en Navarra, C. Valenciana, Galicia y Cataiuhla +infoCLICK EN LA ...

infolweet aernet es

\section{5.- Referencias}

- AEMET 2015 versión 6 Plan Nacional de Predicción y Vigilancia de Fenómenos Meteorológicos Adversos

- AEMET 2017 versión 5 Procedimiento del Plan Nacional de Predicción y Vigilancia de Fenómenos Meteorológicos Adversos

- BOE 14/02/2008 Real Decreto 186/2008

\section{- www.aemet.es}

